\title{
LncRNA AWPPH promotes the proliferation, migration and invasion of ovarian carcinoma cells via activation of the Wnt/ $\beta$-catenin signaling pathway
}

\author{
GUANGYU YU ${ }^{1 *}$, WENSHUANG WANG ${ }^{2 *}$, JUNFENG DENG $^{1}$ and SHAOHUA DONG ${ }^{3}$ \\ ${ }^{1}$ Department of Obstetrics and Gynecology, Yantai Hospital of Traditional Chinese Medicine; \\ ${ }^{2}$ Department of Gynecology, The Affiliated Yantai Yuhuangding Hospital of Qingdao University; \\ ${ }^{3}$ Department of Gynecology, Yantai Yeda Hospital, Yantai, Shandong 264000, P.R. China
}

Received May 20, 2018; Accepted February 8, 2019

DOI: $10.3892 / \mathrm{mmr} .2019 .10029$

\begin{abstract}
The oncogenic role of the long noncoding RNA associated with poor prognosis of hepatocellular carcinoma (lncRNA AWPPH) was reported in various types of malignancies; however, its involvement in ovarian carcinoma (OC) remains unknown. Thus, the present study investigated the role of AWPPH in OC. The expression of AWPPH in tissues and serum acquired from patients with $\mathrm{OC}$, and healthy controls, was determined via reverse transcription-quantitative polymerase chain reaction. The diagnostic value of serum AWPPH expression was evaluated by receiver operating characteristic curve analysis. Additionally, survival curve analysis was performed to determine the prognostic value of AWPPH for OC. An AWPPH overexpression vector was transfected into OC cell lines. Cell proliferation, migration and invasion were analyzed via Cell Counting Kit-8, Transwell migration and invasion assays, respectively. The expression of $\beta$-catenin was investigated via western blotting. It was revealed that the expression levels of AWPPH were significantly upregulated in OC tissues and serum compared with healthy controls. The serum levels of AWPPH were able to effectively diagnose and predict the prognosis of patients with OC. AWPPH overexpression promoted the proliferation, migration and invasion of OC cells, and upregulated $\beta$-catenin expression. Treatment with a Wnt agonist markedly altered AWPPH expression; however, inhibition of Wnt suppressed the effects of AWPPH overexpression on proliferation, migration and invasion of OC
\end{abstract}

Correspondence to: Dr Shaohua Dong, Department of Gynecology, Yantai Yeda Hospital, 11 Taishan Road, Yantai, Shandong 264000, P.R. China

E-mail: skrgnx9@163.com

*Contributed equally

Key words: ovarian carcinoma, long noncoding RNA, AWPPH, $\mathrm{Wnt} / \beta$-catenin signaling pathway, proliferation, migration, invasion cells. Therefore, it was revealed that AWPPH may promote OC via activation of the $\mathrm{Wnt} / \beta$-catenin signaling pathway.

\section{Introduction}

Ovarian carcinoma $(\mathrm{OC})$ is a type of malignancy that originates in the ovaries, and annually affects $>200,000$ individuals and leads to $>140,000$ cases of OC-associated mortality in females worldwide (1). Screening is widely used in the early diagnosis of OC; however, at present, the majority of screening methods have been demonstrated to be ineffective $(2,3)$. The survival of patients with OC markedly improved following treatment with chemotherapy, olaparib maintenance therapy and radiation therapy $(4,5)$; however, no additional improvements in patient survival were observed in subsequent decades (6). There is a lack of clear symptoms during the early stages of $\mathrm{OC}$; therefore, the majority of patients with $\mathrm{OC}$ are diagnosed at advanced stages with metastasis, leading to high mortality rates (7). At present, early diagnosis and treatment remains critical for the survival of patients with OC.

The Wnt/ $\beta$-catenin pathway serves an important role in the onset, development and progression of numerous types of tumors, including OC (8). Activation of Wnt/ $\beta$-catenin signaling in epithelial ovarian cancer regulates the expression of genes involved in cell apoptosis and proliferation, thereby promoting the induction and progression of cancer (8). Long noncoding RNAs (lncRNAs) are a subgroup of noncoding RNAs comprising $>200$ nucleotides, a number of which are involved in the pathogenesis of various types of malignancies via interactions with the $\mathrm{Wnt} / \beta$-catenin pathway $(9,10)$. The IncRNA HOXA transcript at the distal tip activates the Wnt/ $\beta$-catenin pathway in osteosarcoma to increase the chemoresistance of cancer cells (9). In non-small cell lung cancer, the lncRNA small nucleolar RNA host gene (SNHG1) promotes the progression of cancer via activation of the Wnt/ $\beta$-catenin signaling pathway (10). Associated with poor prognosis of hepatocellular carcinoma (AWPPH) is a novel lncRNA that serves an oncogenic role in hepatocellular carcinoma (11) and bladder cancer (12). In the present study, the role of AWPPH in OC was investigated and it was observed that the lncRNA was upregulated in OC; AWPPH may serve 
to promote $\mathrm{OC}$ via activation of the $\mathrm{Wnt} / \beta$-catenin signaling pathway.

\section{Materials and methods}

Specimens. The present study was a retrospective analysis. Tumor and adjacent healthy tissues within $2 \mathrm{~cm}$ of the tumor were collected from 58 patients with OC. Blood was extracted from the 58 patients and stored at room temperature for $2 \mathrm{~h}$, followed by centrifugation at $1,000 \mathrm{x}$ g for $20 \mathrm{~min}$ at $4{ }^{\circ} \mathrm{C}$ to collect supernatant for serum analysis. Patients were treated at Yantai Yeda Hospital (Yantai, China) from June 2011 to June 2012. The age of patients ranged from 30-69 years, with a mean age of $49.4 \pm 6.3$ years. Inclusion criteria for the enrolment of patients were as follows: i) Patients were pathologically diagnosed with OC; ii) patients were initially diagnosed and treated at Yantai Yeda Hospital; iii) clinical data was collected from patients; and iv) patients completed follow-up care. Exclusion criteria were as follows: i) Patients possessed a history of other malignancies; ii) patients exhibited additional types of ovarian diseases; iii) patients were treated prior to admission; and iv) patients succumbed to mortality due to separate diseases during follow-up. All patients possessed epithelial tumors. According to the American Joint Committee on Cancer staging system (13), there were 6 cases in stage II, 8 in stage III and 44 in stage IV.

Additionally, serum samples were obtained from 46 healthy individuals that received routine physiological examinations at Yantai Yeda Hospital during the aforementioned time period to serve as the control group. Controls were enrolled to match the distributions of age and gender of cancer patients. The age of healthy controls ranged from 33 to 69 years, with a mean age of $49.9 \pm 6.1$ years. No significant differences in age were identified between the two groups. All patients and healthy controls signed informed consent forms, and the study was approved by the Ethics Committee of Yantai Yeda Hospital.

Cell culture and transfection. A total of two human OC cell lines, UWB1.289 (CRL-2945 ${ }^{\mathrm{TM}}$ ) and UWB1.289 + BRCA1 (CRL-2946 ${ }^{\mathrm{TM}}$ ), were obtained from the American Type Culture Collection (ATCC; Manassas, VA, USA). Cells were cultured with 50\% ATCC-formulated RPMI-1640 medium and 50\% Mammary Epithelial Cell Growth medium (ATCC) supplemented with $3 \%$ fetal bovine serum (ATCC) in an incubator $\left(37^{\circ} \mathrm{C}, 5 \% \mathrm{CO}_{2}\right)$.

Full-length AWPPH cDNA (Accession: NR_015395.2, Sangon Biotech Co., Ltd., Shanghai, China) was obtained via polymerase chain reaction (PCR) and inserted into a pIRSE2-EGFP vector (Clontech Laboratories, Inc., Mountainview, CA, USA). The restriction sites were BamH I and EcoR I. Empty vector was used as the negative control (NC). AWPPH overexpression vectors were transfected into $4 \times 10^{5}$ cells at a dose of $10 \mathrm{nmol}$ using Lipofectamine ${ }^{\circledR} 2000$ reagent (Invitrogen; Thermo Fisher Scientific, Inc., Waltham, MA, USA). Cells were cultured in an incubator $\left(37^{\circ} \mathrm{C}, 5 \% \mathrm{CO}_{2}\right)$ following transfection, and were collected at $24 \mathrm{~h}$ after transfection. The expression levels of AWPPH were determined via reverse transcription-quantitative PCR (RT-qPCR), and an overexpression rate of $150-200 \%$ was achieved prior to subsequent experimentation. Control group was non-transfected cells and cells transfected with the negative control vector were negative control cells.

For Wnt Agonist treatment, $5 \times 10^{5}$ cells were incubated with 10 ng/ml Wnt Agonist (CAS\# 853220-52-7; Santa Cruz Biotechnology Inc., Dallas, TX, USA) at $37^{\circ} \mathrm{C}\left(5 \% \mathrm{CO}_{2}\right)$ for $12 \mathrm{~h}$ prior to experimentation. For Wnt inhibitor treatment, $2.5 \mu \mathrm{mol}$ inhibitor of Wnt production 2 (IWP-2; SigmaAldrich; Merck KGaA, Darmstadt, Germany) was added into culture medium containing $5 \times 10^{5}$ cells $\left(37^{\circ} \mathrm{C}, 5 \% \mathrm{CO}_{2}\right)$ and incubated for $12 \mathrm{~h}$ prior to experimentation; $1,000 \mathrm{X}$ stocks of Wnt Agonist and IWP-2 were prepared in culture medium.

Cell Counting Kit-8 (CCK-8) assay. Cell proliferation was determined via a CCK-8 assay. Briefly, a 96-well plate was seeded with $100 \mu \mathrm{l}$ cells of UWB1.289 and UWB1.289 + BRCA1 cell lines suspended in a medium of $50 \%$ ATCC-formulated RPMI-1640 medium and 50\% Mammary Epithelial Cell Growth medium supplemented with 3\% fetal bovine serum at a density of $4 \times 10^{3}$ cells/well. Cell culture was performed in an incubator $\left(37^{\circ} \mathrm{C}, 5 \% \mathrm{CO}_{2}\right)$, and $10 \mu \mathrm{CCK}-8$ solution (Sigma-Aldrich; Merck KGaA) was added to each well 24, 48, 72 and $96 \mathrm{~h}$ later. Cells were incubated for an additional $4 \mathrm{~h}$ at $37^{\circ} \mathrm{C}$, and the optical density $(450 \mathrm{~nm})$ was measured using a Fisherbrand ${ }^{\mathrm{TM}}$ accuSkan $^{\mathrm{TM}}$ GO UV/Vis Microplate Spectrophotometer (Fisher Scientific; Thermo Fisher Scientific, Inc.).

Transwell migration and invasion assay. Transwell migration assays were performed by plating $4 \times 10^{3}$ cells in the upper chamber of Transwell plates in $0.1 \mathrm{ml}$ serum-free culture medium, and RPMI-1640 medium (Thermo Fisher Scientific, Inc.) containing 20\% fetal calf serum (Sigma-Aldrich; Merck $\mathrm{KGaA}$ ) was added to the lower chamber. Cells were incubated at $37^{\circ} \mathrm{C}$ for $24 \mathrm{~h}$, and membranes were collected and stained with $0.5 \%$ crystal violet (Sigma-Aldrich; Merck KGaA) for 15 min at room temperature. Cells were observed under an optical microscope and 5 visual fields (magnification, $\mathrm{x} 40$ ) were selected from each membrane to count cell number. For invasion assays, the upper chamber was coated with Matrigel ${ }^{\circledR}$ (Merck KGaA), with all other steps performed as previously described.

$R T-q P C R$. Total RNA was extracted from tissues that were ground in liquid nitrogen using TRIzol ${ }^{\circledR}$ reagent (Invitrogen; Thermo Fisher Scientific, Inc.). Total RNA was also extracted from OC cells. cDNA was synthesized via RT using SuperScript III Reverse Transcriptase kit (Thermo Fisher Scientific, Inc.) with the following conditions: $25^{\circ} \mathrm{C}$ for $5 \mathrm{~min}, 55^{\circ} \mathrm{C}$ for $10 \mathrm{~min}$ and $80^{\circ} \mathrm{C}$ for $5 \mathrm{~min}$. qPCR was performed using SYBR ${ }^{\circledR}$ Green Real-Time PCR Master Mixes (Thermo Fisher Scientific, Inc.). The following primer pairs were used: AWPPH, forward 5'-CTGGATGGTCGCTGCTTTTTA-3', reverse, 5'-AGG GGGATGAGTCGTGATTT-3'; and $\beta$-actin, forward 5'-GAC CTCTATGCCAACACAGT-3' and reverse, 5'-AGTACTTGC GCTCAGGAGGA-3'. The following thermocycling conditions were used for PCR: $40 \mathrm{sec}$ at $95^{\circ} \mathrm{C}$, followed by 40 cycles at $95^{\circ} \mathrm{C}$ for $12 \mathrm{sec}$ and $60^{\circ} \mathrm{C}$ for $40 \mathrm{sec}$. The relative expression levels of AWPPH were normalized to $\beta$-actin using the $2^{-\Delta \Delta C q}$ method (14). 


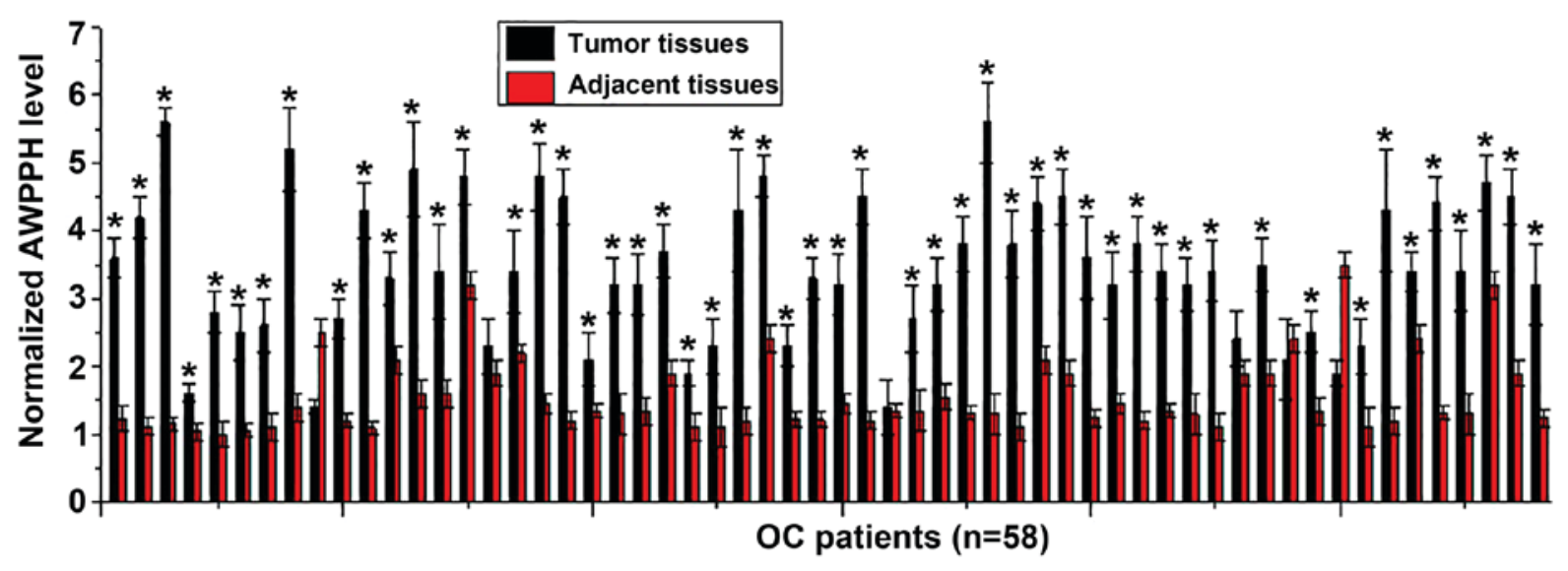

Figure 1. Expression of 1ncRNA AWPPH in tumor tissues and adjacent healthy tissues of patients with OC. Expression levels of 1ncRNA AWPPH in tumor and adjacent healthy tissues of 58 patients with $\mathrm{OC}$ as determined by reverse transcription-quantitative polymerase chain reaction. The expression levels of IncRNA AWPPH were significantly increased in the majority of tumor tissues compared with in the adjacent healthy tissues. The experiment was performed in triplicate. Data are presented as the mean \pm standard deviation. " $\mathrm{P}<0.05$ vs. healthy control tissue. LncRNA AWPPH, long noncoding RNA associated with poor prognosis of hepatocellular carcinoma; OC, ovarian carcinoma.

Western blotting. Radioimmunoprecipitation assay solution (Thermo Fisher Scientific, Inc.) was used to extract total protein from in vitro cultured OC cells. according to the manufacturer's protocols. A bicinchoninic acid assay was performed to determine protein concentration. Proteins (20 $\mu \mathrm{g}$ /lane) were separated via 10\% SDS-PAGE. Proteins were transferred to polyvinylidene difluoride membranes, which were incubated with $5 \%$ skimmed milk at room temperature for $1 \mathrm{~h}$ for blocking. Membranes were then incubated with rabbit anti- $\beta$-catenin antibody $(1: 1,200$; ab6302, Abcam, Cambridge, UK) and anti-GAPDH primary antibody $(1: 1,400$; ab8245, Abcam) overnight at $4^{\circ} \mathrm{C}$, followed by incubation with a horseradish peroxidase-conjugated anti-rabbit IgG-HRP secondary antibody (1:1,000; MBS435036, MyBioSource, Inc., San Diego, CA, USA) at room temperature for $4 \mathrm{~h}$. An enhanced chemiluminescence kit (Sigma-Aldrich; Merck $\mathrm{KGaA}$ ) was then applied to visualize the bands. Membranes were scanned using a MYECL ${ }^{\text {TM }}$ Imager (Thermo Fisher Scientific, Inc.), and $\beta$-catenin expression was normalized to GAPDH expression using Image J V 1.6 software (National Institutes of Health, Bethesda, MD, USA).

Statistical analysis. Each experiment was performed on 3 biological replicates. SPSS 19.0 (IBM Corp., Armonk, NY, USA) was used for all statistical analyses. Data were presented as the mean \pm standard deviation. The diagnostic value of serum AWPPH for OC was investigated by receiver operating characteristic (ROC) curve analysis. Associations between the serum levels of AWPPH and the clinicopathological data of patients with $\mathrm{OC}$ were analyzed using $\chi^{2}$ tests. Comparisons between two groups and across $>2$ groups were performed by unpaired t-tests and one-way analyses of variance followed by post hoc least significant difference tests, respectively. The 58 patients with OC were divided into high- $(n=27)$ and low- $(\mathrm{n}=31)$ AWPPH expression groups according to Youden's index (13). Kaplan-Meier analysis was performed to determine the survival of patients in the two groups, and a log rank test was used to compare survival curves. $\mathrm{P}<0.05$ was considered to indicate a statistically significant difference.

\section{Results}

Expression of IncRNA AWPPH in tumor and adjacent healthy tissues of patients with $O C$. The expression levels of lncRNA AWPPH in tumor and adjacent healthy tissues of 58 patients with OC were determined by RT-qPCR. As presented in Fig. 1, significantly increased expression of AWPPH in tumor tissues compared with in adjacent healthy tissues was observed in $89.7 \%$ (52/58) of patients with OC. The data suggested that upregulation of AWPPH may be involved in the pathogenesis of OC.

Serum levels of AWPPH in patients with OC and healthy controls, and the diagnostic and prognostic values. The serum expression levels of AWPPH in patients with OC and healthy controls were determined by RT-qPCR. As presented in Fig. 2A, the expression levels of AWPPH were significantly increased in the serum acquired from patients with OC compared with in healthy controls $(\mathrm{P}<0.05)$. The diagnostic value of serum AWPPH for OC was investigated by ROC curve analysis. As presented in Fig. 2B, the area under the curve was 0.9082 , with a standard error value of 0.9082 and $95 \%$ confidence interval from 0.8543 to 0.9620 . The 58 patients with OC were divided into high- $(n=27)$ and low- (n=31) AWPPH expression groups according to Youden's index (15). Kaplan-Meier analysis was performed to determine the survival of patients in the two groups, and a log rank test was used to compare survival curves. As presented in Fig. 2C, the overall survival rate of patients with low serum levels of AWPPH was significantly higher compared with patients with high AWPPH serum levels (log rank test $\mathrm{P}=0.0322$ ).

$\chi^{2}$ analysis of the associations between the serum levels of AWPPH and the clinicopathological data of patients. Patients were divided into high- $(\mathrm{n}=29)$ and low-expression $(n=29)$ groups according to the median value of expression. A $\chi^{2}$ test was performed to analyze the associations between the serum levels of AWPPH and the clinicopathological data of patients with OC. As presented in Table I, the serum levels 
A

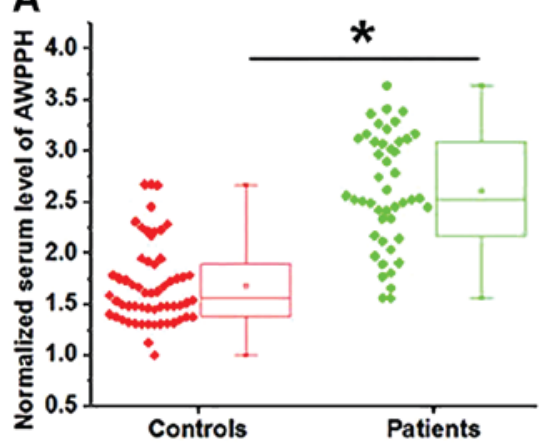

B

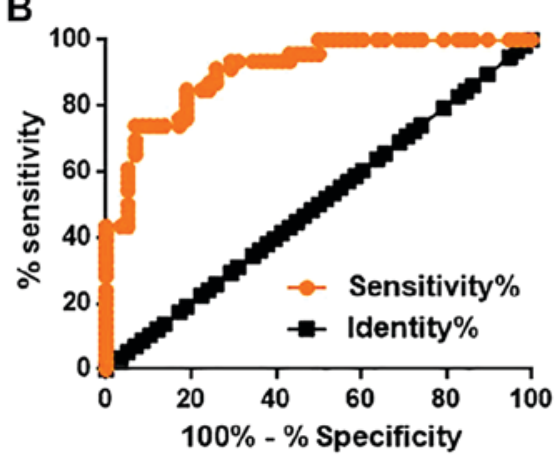

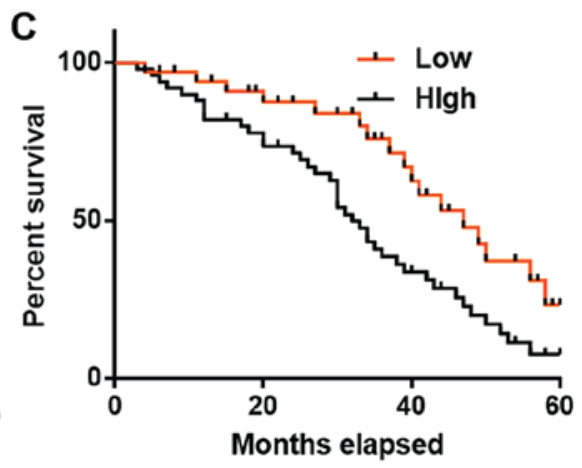

Figure 2. Serum levels of AWPPH in patients with OC and healthy controls, and diagnostic and prognostic values. (A) Comparison of serum levels of AWPPH between patients with OC and healthy controls. (B) Receiver operating characteristic curve analysis of the diagnostic value of serum AWPPH for OC. (C) Comparison of survival curves between patients with high and low serum levels of AWPPH. The serum levels of AWPPH were determined by reverse transcription-quantitative polymerase chain reaction analysis performed in triplicate. Data are presented as the mean \pm standard deviation. $\mathrm{P}<0.05$. AWPPH, associated with poor prognosis of hepatocellular carcinoma; OC, ovarian carcinoma.

of AWPPH were not significantly associated with the age, or drinking and smoking habits of patients; however, the serum levels of lncRNA AWPPH exhibited a significant association with tumor size and tumor distant metastasis.

Effects of AWPPH overexpression on $\beta$-catenin expression. The clinicopathological data presented in Table I indicated that AWPPH may be involved in the regulation of tumor growth and metastasis of OC. Wnt/ $\beta$-catenin serves important roles in the progression of various types of malignancies, such as ovarian cancer (8). In the present study, AWPPH overexpression was induced via transfection with a pIRSE2-EGFP plasmid containing AWPPH cDNA (Fig. 3A). Transfection significantly promoted the expression of $\beta$-catenin in two human OC cell lines, UWB1.289 and UWB1.289 + BRCA1, compared with the control groups of non-transfected cells and cells transfected with the $\mathrm{NC}$ vector $(\mathrm{P}<0.05$; Fig. $3 \mathrm{~B})$. Conversely, treatment with $10 \mathrm{ng} / \mathrm{ml}$ Wnt Agonist did not significantly affect AWPPH expression ( $\mathrm{P}>0.05$; data not shown).

Effects of AWPPH overexpression and Wht inhibitor on cell proliferation, migration and invasion. As presented in Fig. 4, AWPPH overexpression significantly promoted cell proliferation, migration and invasion of the two human OC cell lines compared with the controls. In addition, treatment with $2.5 \mu \mathrm{mol}$ IWP-2 significantly reduced the enhancing effects of AWPPH overexpression on each cellular property. The data suggested that AWPPH may promote cell proliferation, migration and invasion in $\mathrm{OC}$ via activation of the $\mathrm{Wnt} / \beta$-catenin pathway.

\section{Discussion}

The key finding of the present study is that lncRNA AWPPH, previously identified as an oncogene in hepatocellular carcinoma (11) and bladder cancer (12), may serve a similar role in OC. The oncogenic effects of AWPPH in OC may be achieved via activation of the Wnt/ $\beta$-catenin signaling pathway. Furthermore, the results revealed that AWPPH may be involved in regulating the growth and metastasis of OC.
The development of OC is accompanied with alterations in the expression profiles of numerous lncRNAs (16). Various lncRNAs exhibit altered expression profiles and serve separate roles in OC to inhibit or promote tumor progression. Decreased expression levels of lncRNA maternally expressed 3 were reported in OC tissues compared with adjacent healthy tissues, and upregulation of this lncRNA suppressed tumor progression (17). Conversely, SNHG1 expression is significantly upregulated in OC tissues, indicating an oncogenic role in OC (18). Upregulation of AWPPH was observed in hepatocellular carcinoma (11) and bladder cancer (12). In the present study, significantly increased levels of AWPPH expression were reported in tumor tissues compared with adjacent healthy tissues in the majority of patients with $\mathrm{OC}$, indicating a potentially oncogenic role for the lncRNA in the pathogenesis of OC.

Tumor metastasis is the main obstacle in the treatment of $\mathrm{OC}$, and early diagnosis and treatment is important for the survival of patients with OC. The onset of disease in humans is usually associated with alterations in the levels of certain substances in the blood, the detection of which may aid diagnosis and improve prognosis of human diseases (19). In the present study, ROC curve analysis revealed that serum AWPPH was able to effectively separate patients with OC from healthy controls. Additionally, increased serum levels of AWPPH were associated with shorter survival time. The serum levels of AWPPH did not correlate with the age, or smoking and drinking habits of patients, which have been demonstrated to affect the expression of certain lncRNAs (19-22). Therefore, AWPPH may serve as a potential diagnostic and prognostic biomarker for OC; however, as a novel lncRNA, the expression profile of AWPPH in other human diseases have not yet been reported. Therefore, the inclusion of additional biomarkers may improve the accuracy of diagnosis and prognosis of patients.

The present study also revealed that the serum levels of AWPPH were associated with distant tumor metastasis and tumor size. The Wnt/ $\beta$-catenin pathway serves important roles in tumor progression in various types of malignancies, including ovarian cancer (8). The results demonstrated that AWPPH overexpression significantly promoted the 
Table I. $\chi^{2}$ analysis of the association between the serum levels of long noncoding RNA associated with poor prognosis of hepatocellular carcinoma and the clinicopathological data of patients.

\begin{tabular}{|c|c|c|c|c|c|c|}
\hline Clinicopathological factor & Groups & Cases & High-expression & Low-expression & $\chi^{2}$ & P-value \\
\hline \multirow[t]{2}{*}{ Age (years) } & $>50$ & 28 & 13 & 15 & \multirow[t]{2}{*}{0.27} & \multirow[t]{2}{*}{0.60} \\
\hline & $<50$ & 30 & 16 & 14 & & \\
\hline \multirow[t]{2}{*}{ Smoking } & Yes & 16 & 7 & 9 & \multirow[t]{2}{*}{0.35} & \multirow[t]{2}{*}{0.56} \\
\hline & No & 42 & 22 & 20 & & \\
\hline \multirow[t]{2}{*}{ Drinking } & Yes & 18 & 8 & 10 & \multirow[t]{2}{*}{0.32} & \multirow[t]{2}{*}{0.57} \\
\hline & No & 40 & 21 & 19 & & \\
\hline \multirow[t]{2}{*}{ Primary tumor diameter } & $>2 \mathrm{~cm}$ & 27 & 19 & 8 & \multirow[t]{2}{*}{8.35} & \multirow[t]{2}{*}{$0.01^{\mathrm{a}}$} \\
\hline & $<2 \mathrm{~cm}$ & 31 & 10 & 21 & & \\
\hline \multirow[t]{2}{*}{ Distant tumor metastasis } & Yes & 34 & 22 & 12 & \multirow[t]{2}{*}{7.11} & \multirow[t]{2}{*}{$0.01^{\mathrm{a}}$} \\
\hline & No & 24 & 7 & 17 & & \\
\hline
\end{tabular}

${ }^{\mathrm{a}} \mathrm{P}<0.05$.
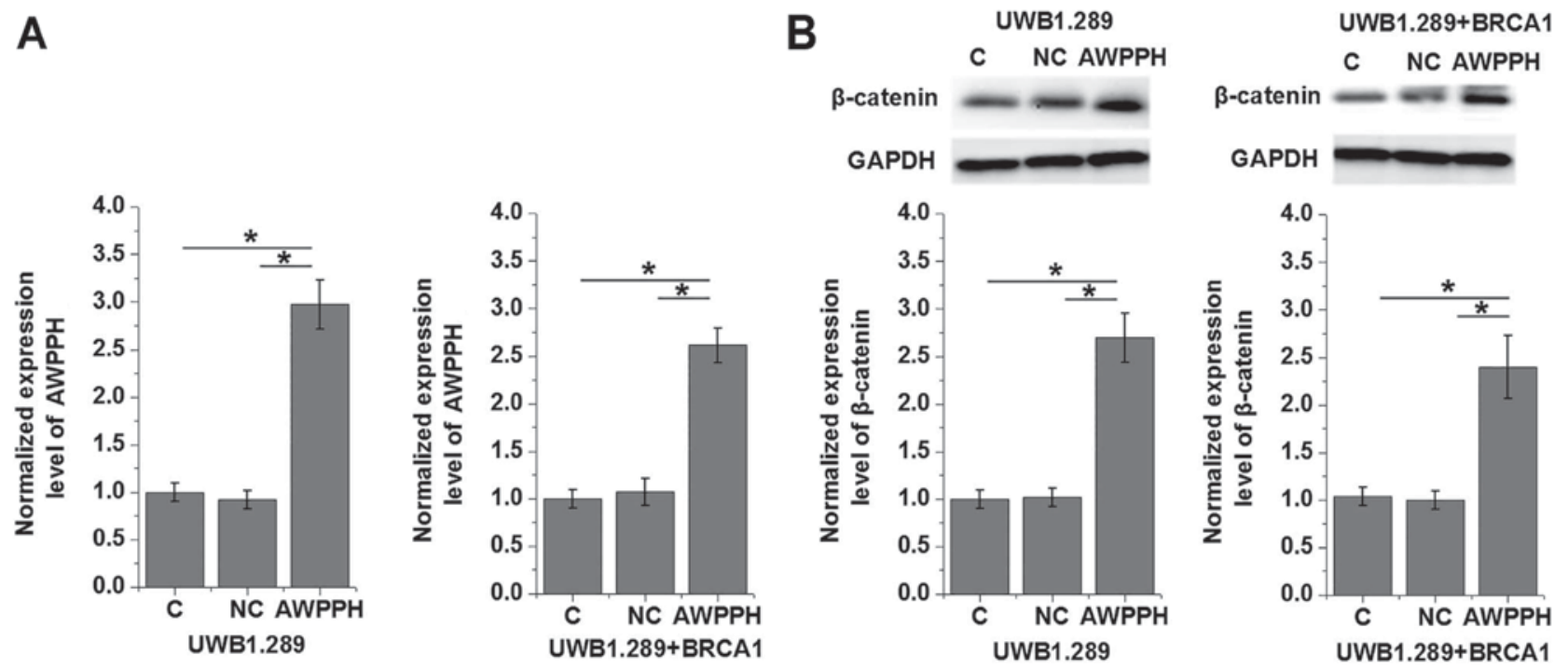

Figure 3. Effects of AWPPH overexpression on $\beta$-catenin expression. Reverse transcription-quantitative polymerase chain reaction and western blot analyses revealed elevated levels of (A) AWPPH mRNA and (B) $\beta$-catenin protein expression in UWB1.289 and UWB1.289 + BRCA1 cells following transfection with AWPPH plasmid, compared with cells transfected with NC vector and non-transfected cells. Western blotting was performed in triplicate. Data are presented as the mean \pm standard deviation. ${ }^{*} \mathrm{P}<0.05$. AWPPH, associated with poor prognosis of hepatocellular carcinoma; NC, negative control vector; OC, ovarian carcinoma.

expression of $\beta$-catenin in two human OC cell lines. Conversely, Wnt $/ \beta$-catenin activation exhibited no significant effects on the expression of AWPPH in the cell lines, indicating that AWPPH may be an upstream activator of the Wnt/ $\beta$-catenin pathway. In vitro cell proliferation, migration and invasion assays demonstrated the potential involvement of AWPPH in the regulation of growth and metastasis in OC. Additionally, treatment with the Wnt/ $\beta$-catenin inhibitor IWP-2 eliminated the effects of AWPPH overexpression in these assays, indicating that the roles of AWPPH in OC may involve the $\mathrm{Wnt} / \beta$-catenin pathway.

There are certain limitations of the present study. A small sample size was employed. Additionally, the expression of $\beta$-catenin was only investigated at the protein level; thus, its expression at the mRNA level in OC cells remains unknown. Furthermore, the expression of other genes involved in the Wnt/ $\beta$-catenin signaling pathway was not investigated. Therefore, the molecular mechanisms underlying the regulatory effects of AWPPH on Wnt/ $\beta$-catenin signaling remain unknown. Further investigation of the components of the $\mathrm{Wnt} / \beta$-catenin signaling pathway is required to provide greater insight into the oncogenic properties of AWPPH.

In conclusion, AWPPH expression was upregulated in OC in the present study. The serum expression levels of AWPPH may serve as a potential diagnostic and prognostic biomarker for OC. AWPPH overexpression promoted the proliferation, migration and invasion of OC cells and upregulated $\beta$-catenin expression. Treatment with Wnt Agonist markedly affected AWPPH expression; however, IWP-2 reduced the effects of AWPPH overexpression on proliferation, migration and 

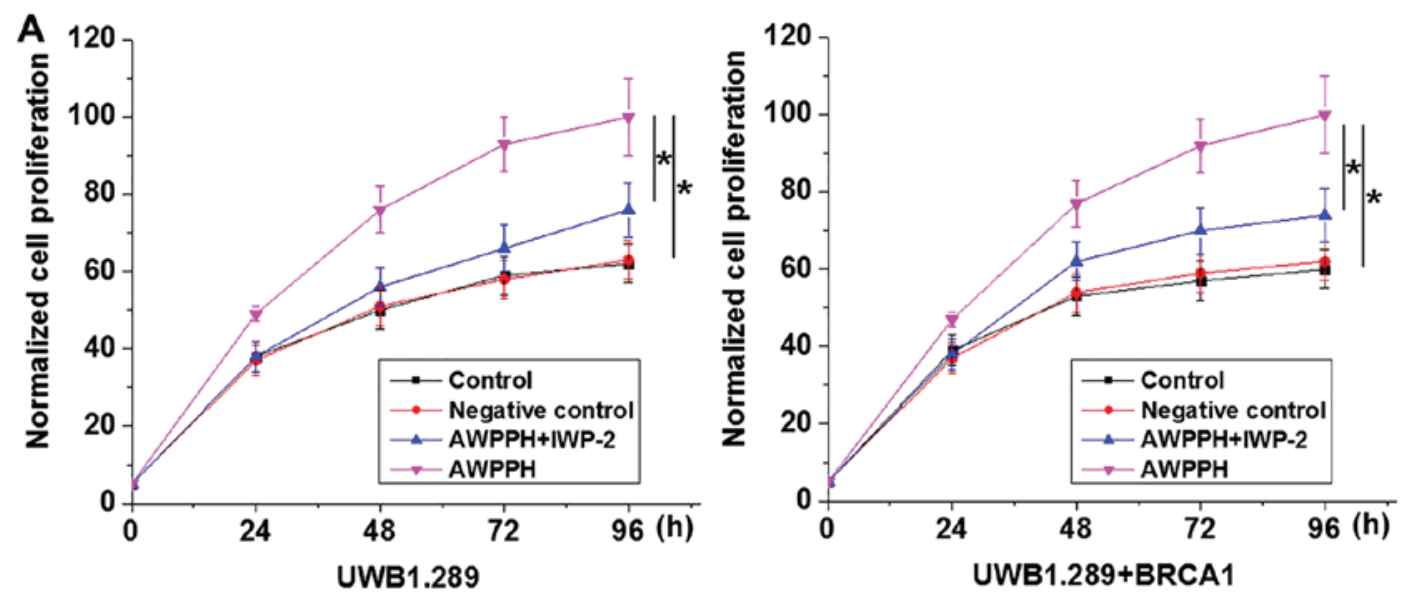

B
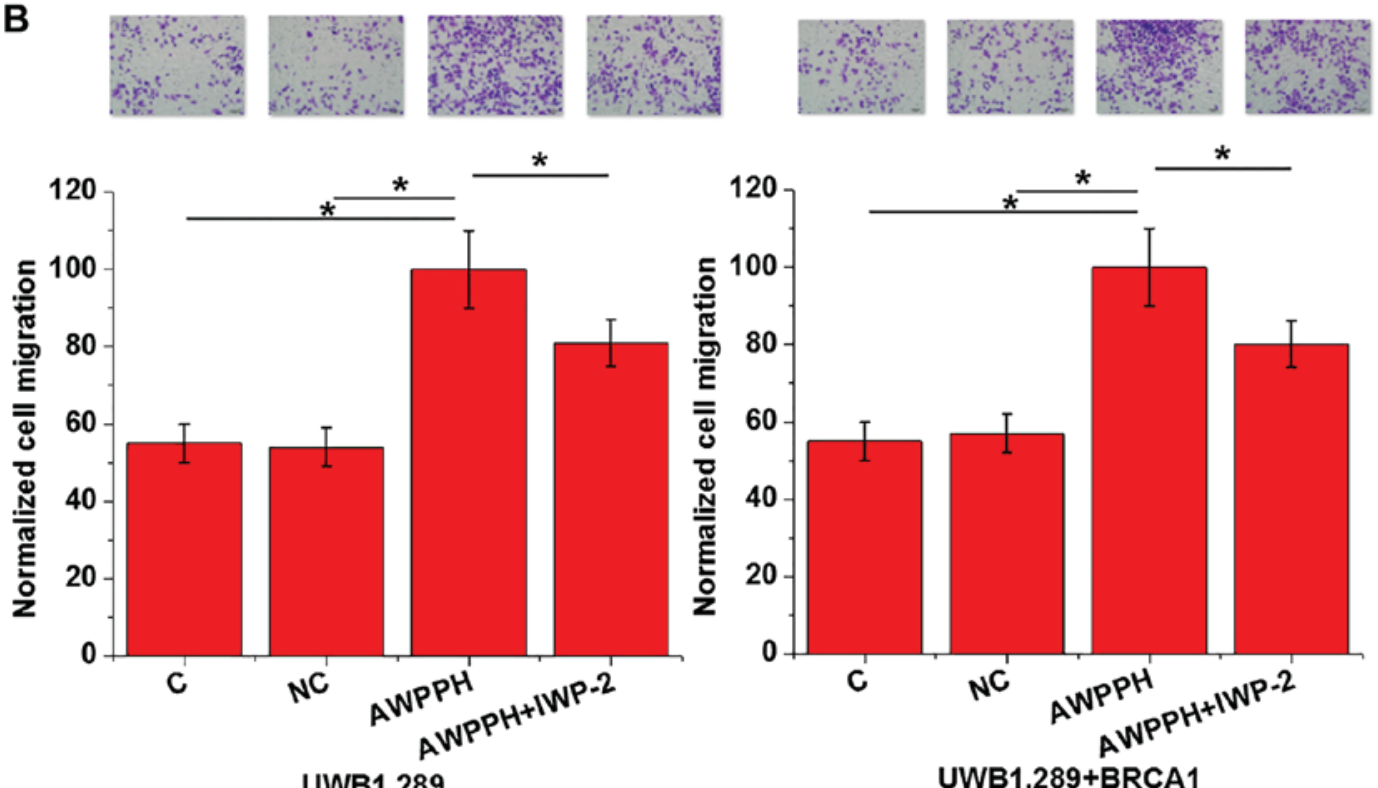

UWB1.289

UWB1.289+BRCA1

C
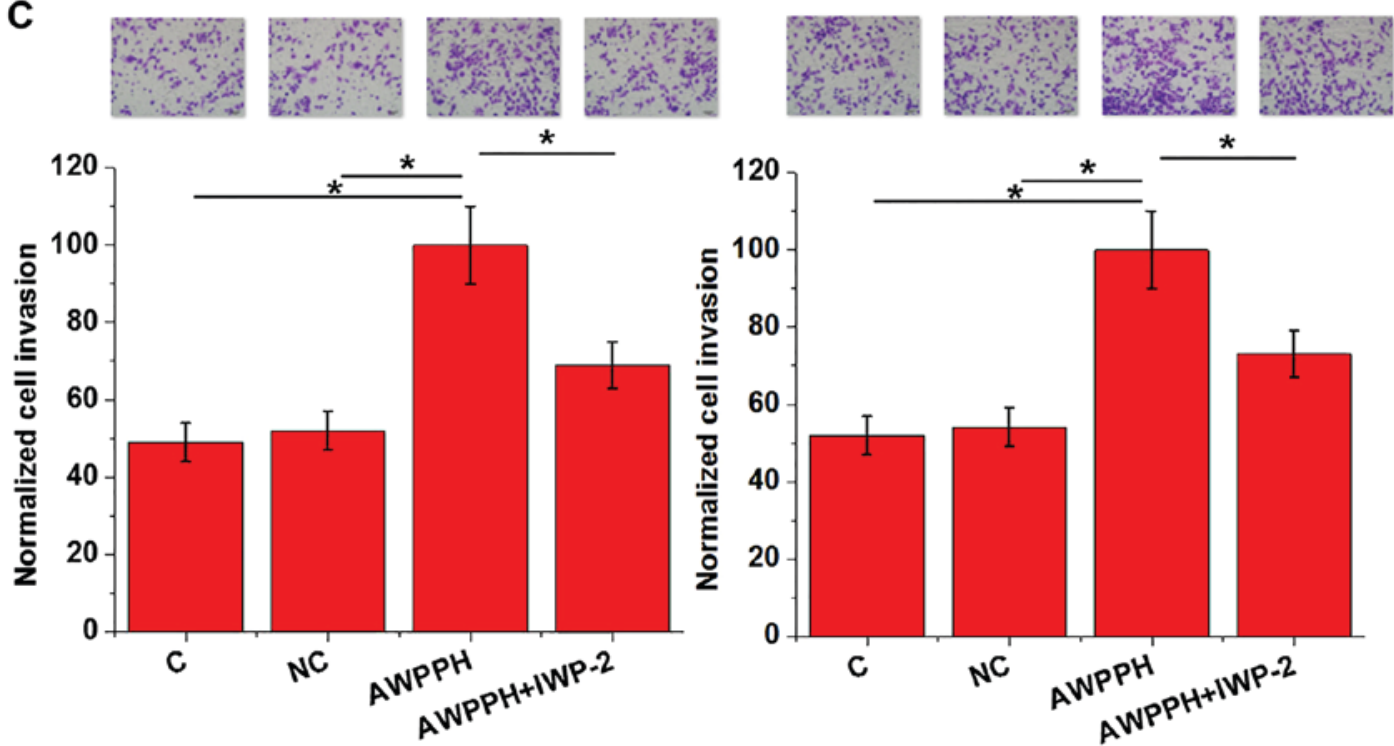

UWB1.289

UWB1.289+BRCA1

Figure 4. Effects of AWPPH overexpression and Wnt inhibition on cell proliferation, migration and invasion. AWPPH overexpression significantly promoted the (A) proliferation, (B) migration and (C) invasion of two human OC cell lines. Treatment with Wnt inhibitor IWP-2 significantly reduced the enhancing effects of AWPPH overexpression. Cell proliferation, migration and invasion assays were performed in triplicate manner. Data are presented as the mean \pm standard deviation. " $\mathrm{P}<0.05$. AWPPH, associated with poor prognosis of hepatocellular carcinoma; $\mathrm{C}$, untreated control; IWP-2, inhibitor of Wnt production 2; NC, negative control. 
invasion of OC cells. Therefore, the results suggested that lncRNA AWPPH may be involved in the pathogenesis of OC, possibly via activation of the $\mathrm{Wnt} / \beta$-catenin signaling pathway.

\section{Acknowledgements}

Not applicable.

\section{Funding}

No funding was received.

\section{Availability of data and materials}

All data generated or analyzed during this study are included in this published article.

\section{Authors' contributions}

GY, WW, JD and SD made substantial contributions to the conception and design of the present study. GY and WW performed the experiments. GY, WW and JD analyzed and interpreted the data. GY and WW drafted the article. GY, WW and SD were responsible for the revision of the manuscript. All authors read and approved the final manuscript.

\section{Ethics approval and consent to participate}

The present study was approved by the Ethics Review Committee of Yantai Yeda Hospital (Yantai, China). All patients provided signed informed consent.

\section{Patient consent for publication}

Not applicable.

\section{Competing interests}

The authors declare that they have no competing interests.

\section{References}

1. Jemal A, Bray F, Center MM, Ferlay J, Ward E and Forman D: Global cancer statistics. CA Cancer J Clin 61: 69-90, 2011.

2. Kobayashi H, Yamada Y, Sado T, Sakata M, Yoshida S, Kawaguchi R, Kanayama S, Shigetomi H, Haruta S, Tsuji Y, et al: A randomized study of screening for ovarian cancer: A multicenter study in Japan. Int J Gynecol Cancer 18: 414-420, 2008.

3. Buys SS, Partridge E, Black A, Johnson CC, Lamerato L, Isaacs C, Reding DJ, Greenlee RT, Yokochi LA, Kessel B, et al: Effect of screening on ovarian cancer mortality: The Prostate, Lung, Colorectal and Ovarian (PLCO) cancer screening randomized controlled trial. JAMA 305: 2295-2303, 2011.

4. Pujade-Lauraine E, Hilpert F, Weber B, Reuss A, Poveda A, Kristensen G, Sorio R, Vergote IB, Witteveen P, Bamias A, et al: AURELIA: A randomized phase III trial evaluating bevacizumab combined with chemotherapy for platinum-resistant recurrent ovarian cancer. J Clin Oncol 30: 327s, 2012.
5. Ledermann J, Harter P, Gourley C, Friedlander M, Vergote I, Rustin G, Scott C, Meier W, Shapira-Frommer R, Safra T, et al: Olaparib maintenance therapy in platinum-sensitive relapsed ovarian cancer. N Engl J Med 366: 1382-1392, 2012.

6. Ovarian Cancer Research Program of BC, Cheryl Brown: Ovarian Cancer Outcomes Unit. OVCARE: Research platforms, 2013. Available at: www.ovcare.ca. Accessed Feb 6, 2014

7. Jayson GC, Kohn EC, Kitchener HC and Ledermann JA: Ovarian cancer. Lancet 384: 1376-1388, 2014.

8. Fodde R and Brabletz T: Wnt/beta-catenin signaling in cancer stemness and malignant behavior. Curr Opin Cell Biol 19: 150-158, 2007.

9. Li Z, Zhao L and Wang Q: Overexpression of long non-coding RNA HOTTIP increases chemoresistance of osteosarcoma cell by activating the Wnt/ $\beta$-catenin pathway. Am J Transl Res 8: 2385-2393, 2016.

10. Cui Y, Zhang F, Zhu C, Geng L, Tian T and Liu H: Upregulated lncRNA SNHG1 contributes to progression of non-small cell lung cancer through inhibition of miR-101-3p and activation of Wnt $/ \beta$-catenin signaling pathway. Oncotarget 8: 17785-17794, 2017.

11. Zhao X, Liu Y and Yu S: Long noncoding RNA AWPPH promotes hepatocellular carcinoma progression through YBX1 and serves as a prognostic biomarker. Biochim Biophys Acta Mol Basis Dis 1863: 1805-1816, 2017.

12. Zhu F, Zhang X, Yu Q, Han G, Diao F, Wu C and Zhang Y: LncRNA AWPPH inhibits SMAD4 via EZH2 to regulate bladder cancer progression. J Cell Biochem 119: 4496-4505, 2018.

13. American Joint Committee on Cancer: Ovary[M]//AJCC cancer staging manual. Springer, New York, NY, pp.275-283, 2002.

14. Livak KJ and Schmittgen TD: Analysis of relative gene expression data using real-time quantitative PCR and the 2(-Delta Delta C(T)) method. Methods 25: 402-408, 2001.

15. Fluss R, Faraggi D and Reiser B: Estimation of the Youden Index and its associated cutoff point. Biom J 47: 458-472, 2005.

16. Zhou M, Sun Y, Sun Y, Xu W, Zhang Z, Zhao H, Zhong Z and Sun J: Comprehensive analysis of lncRNA expression profiles reveals a novel lncRNA signature to discriminate nonequivalent outcomes in patients with ovarian cancer. Oncotarget 7: 32433-32448, 2016.

17. Xiu YL, Sun KX, Chen X, Chen S, Zhao Y, Guo QG and Zong ZH: Upregulation of the lncRNA Meg3 induces autophagy to inhibit tumorigenesis and progression of epithelial ovarian carcinoma by regulating activity of ATG3. Oncotarget 8: 31714-31725, 2017.

18. Zhang M, Wang W, Li T, Yu X, Zhu Y, Ding F, Li D and Yang T: Long noncoding RNA SNHG1 predicts a poor prognosis and promotes hepatocellular carcinoma tumorigenesis. Biomed Pharmacother 80: 73-79, 2016.

19. Moore RG, Brown AK, Miller MC, Skates S, Allard WJ, Verch T, Steinhoff M, Messerlian G, DiSilvestro P, Granai CO and Bast RC Jr: The use of multiple novel tumor biomarkers for the detection of ovarian carcinoma in patients with a pelvic mass. Gynecol Oncol 108: 402-408, 2008.

20. Grammatikakis I, Panda AC, Abdelmohsen K and Gorospe M: Long noncoding RNAs(lncRNAs) and the molecular hallmarks of aging. Aging (Albany NY) 6: 992-1009, 2014

21. Wang J, Qiu M, Xu Y, Li M, Dong G, Mao Q, Yin R and $\mathrm{Xu}$ L: Long noncoding RNA CCAT2 correlates with smoking in esophageal squamous cell carcinoma. Tumour Biol 36: 5523-5528, 2015.

22. Mayfield RD: Emerging roles for ncRNAs in alcohol use disorders. Alcohol 60: 31-39, 2017. International (CC BY-NC-ND 4.0) License. 